\title{
Design and Realization of a Polymer Compliant Stage with 3D Printing and Rubber Damping
}

\author{
Yi-Hsuan $\mathrm{Yu}^{\mathrm{a} 1^{*}}$, Yen-Feng Cheng ${ }^{\mathrm{a} 1}$, Kuo-Shen Chen ${ }^{\mathrm{b} 1}$, Yun-Hui Liu ${ }^{\mathrm{b} 2}$, and Wei-Chin Huang ${ }^{\mathrm{c} 3}$ \\ ${ }^{\mathrm{a}}$ Graduate Student, ${ }^{\mathrm{b}}$ Professor, ${ }^{\mathrm{C}} \mathrm{Ph}$. D. Engineer \\ ${ }^{1}$ Department of Mechanical Engineering, National Cheng-Kung University, \\ No. 1, Daxue Rd., East Dist., Tainan City 701, Taiwan (R.O.C.) \\ ${ }^{2}$ Department of Mechanical Engineering, Southern Taiwan University of Science and Technology, \\ No. 1, Nantai St., Yungkang Dist., Tainan 71005, Taiwan (R.O.C.) \\ ${ }^{3}$ Industrial Technology Research Institute, \\ No. 8, Gongyan Rd., Liujia Dist., Tainan City 73445, Taiwan (R.O.C.) \\ *Corresponding Author: sr77635@hotmail.com
}

\begin{abstract}
Compliant stages are critical subsystems in precision motion control applications. Traditionally, they are usually fabricated by using conventionally machining methods such as line cutting of metallic structures. Although such an approach could yield excellent performance in many applications, it suffers from two major concerns, i.e., weight concern and low material damping, which represent major bottlenecks for further performance improvements. By incorporating with 3D printing technology, it is possible to carry out light weight design for significantly reducing the weight while maintaining the required stiffness. Meanwhile, by further introducing shear damper-liked concept such as liquid elastomer materials in key locations, it is possible to improve the structural damping capability. In this work, a well-recognized compliant stage, served as the platform, is designed and realized by using plastic 3D printing and liquid PDMS casting for investigating the feasibility of the above concept. Essential performance and dynamic tests are conducted to examine the functional performance and dynamic characteristics of the stage. The preliminary characterization results indicate that with proper design, the functional performance of the plastic stage can actually comparable with those of line cut metallic stages. Meanwhile, the enhancement of damping strongly depends on rubber injected position. Rubbers must be added in the portions where strong elastic deformations are expected for improving the damping capability. Although primitive, the
\end{abstract}

study shows promising results for continuing future more comprehensive studies in future stage designs using both plastic and metallic 3D printings.

Keywords: Compliant stages, 3D Printing, Rubbers, Damping.

\section{Introduction}

Compliant stages are critical subsystems in precision motion control for various industrial applications ranged from machining tool handling to sub-micron metrology $y^{(1)}$. Traditionally, they are usually fabricated by using conventionally machining methods such as line cutting of metallic structures ${ }^{(2)}$. Although such an approach could yield excellent performance in many applications, it suffers from two major concerns, i.e., weight concern and low material damping, which represent major bottlenecks for further performance improvements. Recently, with the progress in metallic three-dimensional printing (3DP) technology, many structures can be fabricated in a layer-based additive manner to form complicated inner and surface morphology ${ }^{(3)}$. Light-weighted and high specific stiffness structures can therefore be realized ${ }^{(4)}$. This certainly offers a new direction in structure design for matching the performance without the limitation in geometry and machining.

However, the damping of metallic structures is extremely low if no external dampers are appended. From precision control perspective, such a low damping system provides less sufficient phase margin in control system 
design and additional differential based controller must be incorporated and this could increase the influence of high frequency noise. Conversely, for a plant with more damping, the associate control system design could be easier and yield better performance. However, many miniaturized precision stages may not allow the integration of external dampers due to space limitation and the entire damping capability relies on the material damping itself. Here we propose a concept to enhance the equivalent material damping by creating metallic/rubber micro composited structure based on 3DP technology for creating channels to fill with liquid elastomers at certain key locations. With proper design, the elastomers can provide additional damping based on classic shear damping theory ${ }^{(5)}$. However, the classic theory is mainly used for highly viscous fluid, not for viscoelastic rubber materials. Therefore, the effectiveness and performance of the proposed method should be verified before detail design.

In order to proof the concept and to examining the effectiveness with minimum costs, plastic 3DP structures are used instead of the final metallic 3DP. In addition, simple steel beam structures by traditional machining are also used for quantizing the effect of the location and amount of rubber in damping enhancement. This allows us for detail parametric study without budget concern. Meanwhile, a well-recognized compliant stage ${ }^{(6)}$, served as the platform, is designed and realized by using plastic 3D printing and liquid PDMS casting for investigating the feasibility of the above concept. Essential performance and dynamic tests are conducted to examine the functional performance and dynamic characteristics of the stage. Fig. 1 outlines the investigation flow of this work. By such an effort, it is hope that a rational design flow for integrating 3DP technology and damping enhancement for high performance stage design can be developed for future applications.

The remainder of this paper presents the investigation on realization the concept in detail. A brief description on the stage design and realization is addressed in Section II and followed by the preliminary dynamic characterization results presented in Section III. Meanwhile, a series of cantilever beam tests for examining the damping capability is shown in Section IV. Subsequently, the preliminary dynamic behavior of stage integrated with rubber is demonstrated in Section $\mathrm{V}$ for elucidating the proposed concept. Finally, Section VI briefly discusses the current results, provides future perspective, and concludes this work.

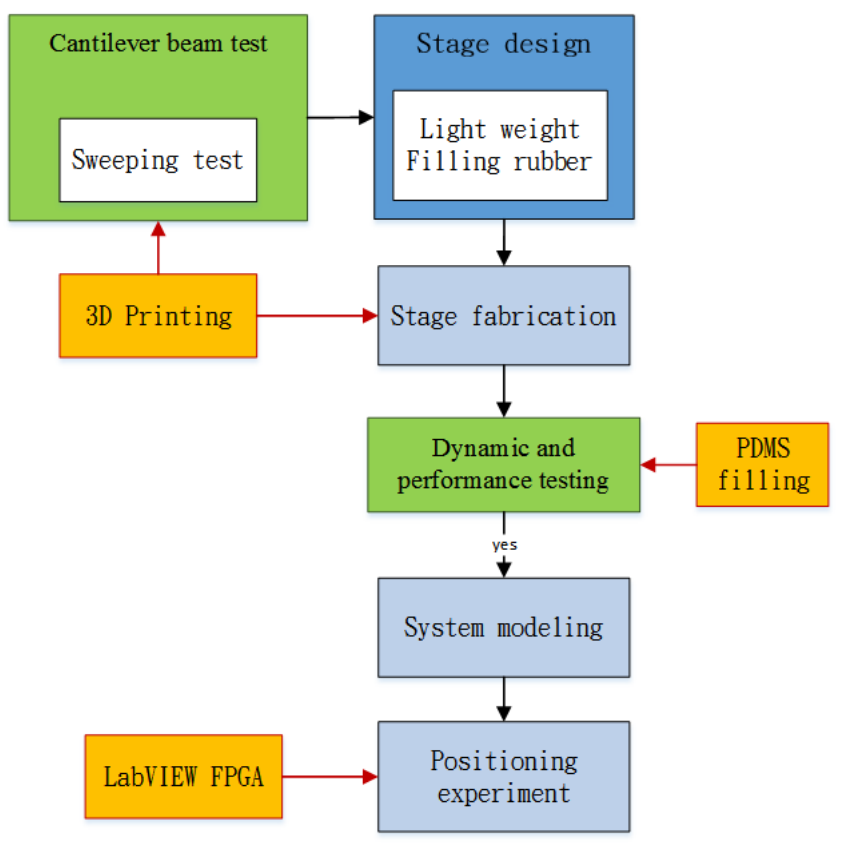

Fig. 1. Research flow for realizing the entire system.

\section{Stage design and realization}

\subsection{Stage design}

A well-defined compliant stage design is adapted in this work for evaluating the proposed performance enhancement strategy. Using a well-defined stage design could provide a more meaningful comparison. Here, we refer to Li's stage design, which is an XY totally decoupled parallel stage (TDPS) $^{(6)}$ shown in Fig. 2. The XY stage consists of four individual limbs connected at the center point B in parallel. Each limb provides essential compliance and serves as a displacement amplification mechanism shown in Fig. 3. This lamb is employed in this work as our main carrier for conducting the study. Li et al. also has conducted numerical analysis about this displacement amplification for a complete compliance analysis ${ }^{(11)}$ based on the potential energy analysis and Castigliano's theorem, the derivation of the amplification ratio, and the input stiffness and output stiffness. Detailed design analysis can be found in ${ }^{(6)}$. Here we adapt the design flow from ${ }^{(6)}$ and the final dimensions are listed in Table 1. From ${ }^{(6)}$, the displacement amplification ratio is estimated as 6.04. Once the geometric design is finished, the structure can be realized by using 3D Printing. 


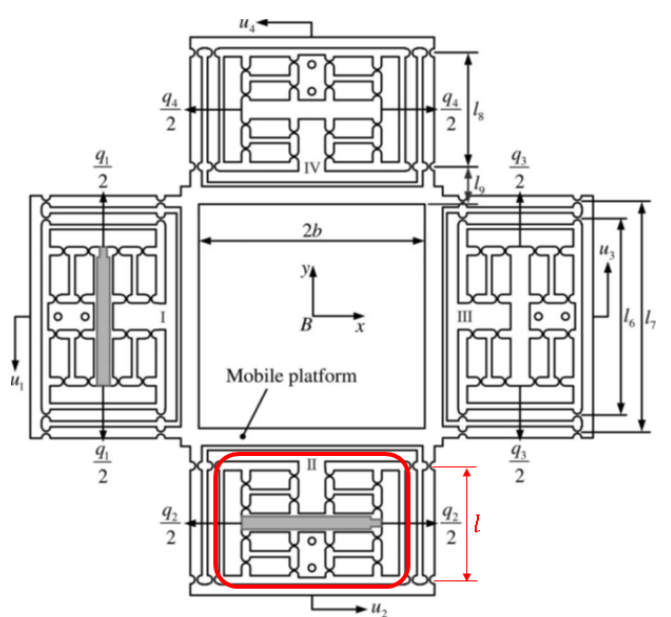

Fig. 2. Symmetric XY TDPS with displacement amplifier ${ }^{(6)}$.

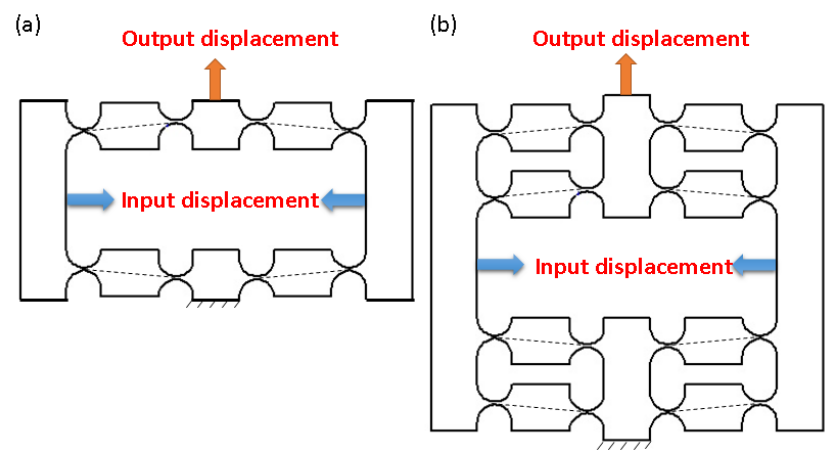

Fig. 3. (a) Original displacement amplifier and (b) an improved displacement amplifier with high transverse stiffness.

Table 1. Parameter of the stage (mm).

\begin{tabular}{|c|c|c|c|c|c|c|}
\hline$w$ & $h_{l}$ & $l_{a}$ & $l_{y}$ & $l_{x}$ & $l_{l}$ & $l_{2}$ \\
\hline 25.4 & 16 & 32 & 22 & 26 & 24 & 47 \\
\hline
\end{tabular}

\subsection{Stage fabrication and rubber filling}

A (model number) 3D printing machine is employed in this work for realizing the stage. The system, with a maximum in-plane working space of $20 \mathrm{~cm} \times 20 \mathrm{~cm}$ and an allowable working height of $40 \mathrm{~cm}$, it has a minimum resolution of $0.3 \mathrm{~mm}$. The material used for constructing the stage is the conventional ABS plastics with a Young's modulus of $2.3 \mathrm{GPa}$ and a Poisson's ratio of 0.35 . Using the commercial 3D printing software Cura, the design file is firstly converted into G-code and the internal filling ratio is set to $20 \%$ for saving the processing cost and create cavities for filling rubbers. Finally, the 3D printers reads the G-code to realize the design. The final structure is shown in Fig. 4(a) for the entire stage and in Fig. 4(b) for a detail illustration on the portion of notch.

In parallel, a finite element analysis performed by ANSYS is also conducted for accessing the structural compliance and to evaluate the state of stress during operation. Based on shear damper theory, damping filling rubbers should be added in the portions where the maximum stress is observed for efficiently dissipating energy. As shown in Fig. 5, the structure at notch hinges have the highest stress level. Therefore, it is expected that rubber materials should be filled into these regimes for maximizing the damping enhancement.
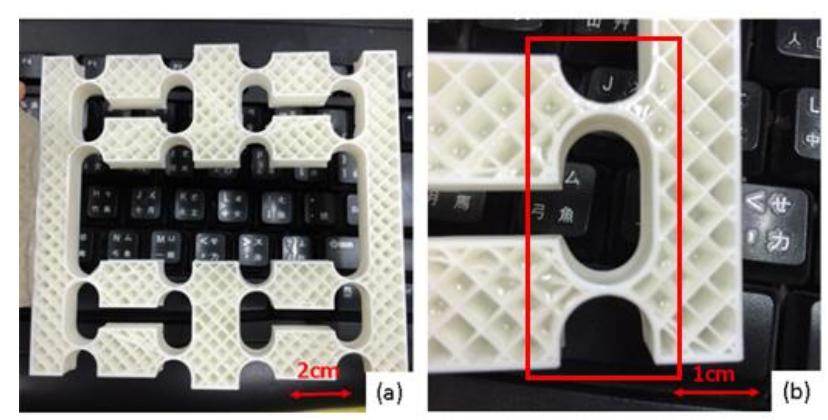

Fig. 4. 3DP polymer compliant stage with a filling ratio of $20 \%$ (a) complete diagram (b) notch hinge

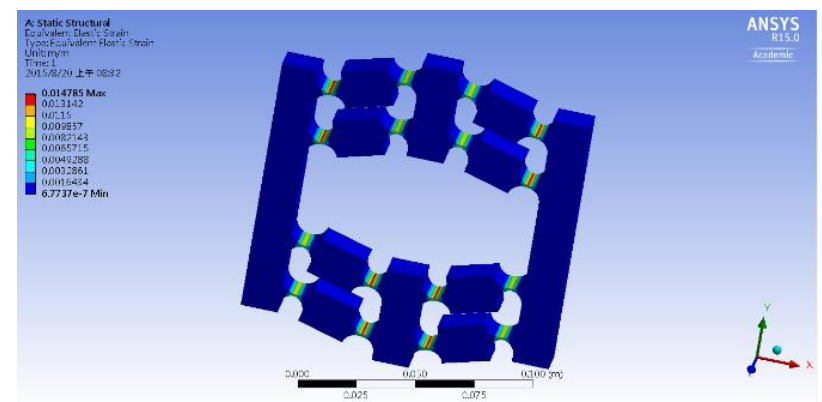

Fig. 5. Deformed finite element model of the compliant stage with $0.5 \mathrm{~mm}$ input displacement.

\section{Dynamic testing and performance evaluation}

\subsection{Dynamic and performance testing setup}

Many compliant stages were actuated by using piezoelectric (PZT) actuators. However, although PZT actuators are in general powerful, their strokes are usually limited and additional amplification mechanisms are usually required. One major advantage of compliant stages made of plastic materials is their low stiffness (the Young's modulus of ABS is approximately $2.3 \mathrm{GPa}$ ) in comparison with that made of metallic materials. This implies that actuators less 
powerful but with a larger stroke are ideally suitable for driving the stage. For this reason, a voice coil motor (VCM) is chosen for this stage. In addition, VCMs are usually have high bandwidth (can up to a few hundred $\mathrm{Hz}$ ). Notice that although the stage is much more compliant than traditional metallic stages, its natural frequency may be still comparable due to its low weight design.

The conceptual layout of the stage is shown in Fig. 6. As mentioned earlier, a VCM (AVM 19-5, 5mm, peak force: $7.79 \mathrm{~N}$ ) is employed. Meanwhile, two laser displacement sensors (YP06MGVL80, $50 \mathrm{~mm}$, resolution $<10 \mu \mathrm{m}$ ) are also used for measurement both the displacements of input and output for determining the amplification ratio. The voice coil pushes the input displacement in sinusoidal manner under different actuating frequencies. A load cell is also attached serially with the VCM for measuring the input force. A picture of the entire setup is shown in Fig.7. Once the setup is ready, the dynamic characterizations of the stage is then followed. The major issues to be examined are the stiffness, natural frequency, and amplification ratio.

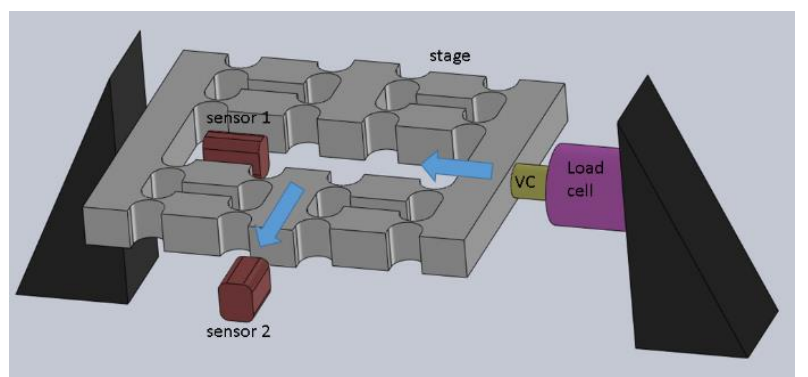

Fig. 6. Schematic plot of the experimental setup.

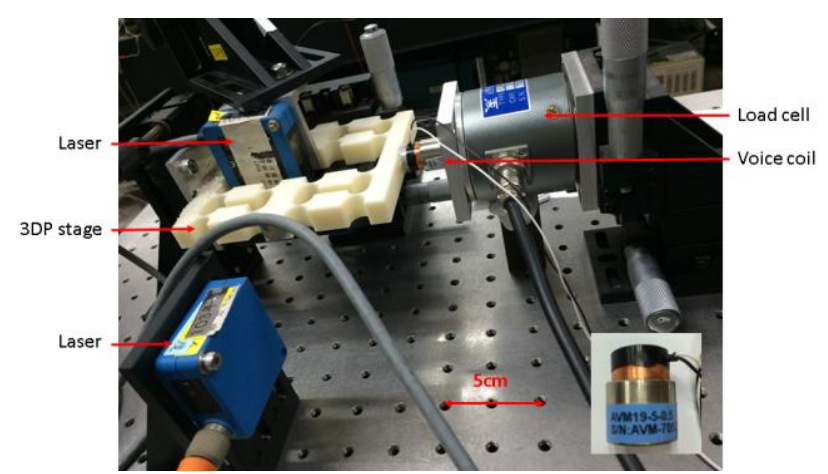

Fig. 7. Experiment system setup.

\subsection{Test result}

Fig. 8 illustrates the loading and displacement histories at $80 \mathrm{~Hz}$ actuation. By systematically performing sinusoidal tests at different frequencies, it is possible to find the frequency responses of the amplification ratios and the stiffness.
The amplification ratios of the compliant stage are shown in Fig. 9. Its value is frequency-dependent. For actuating frequency less than $85 \mathrm{~Hz}$, the average value of amplification ratio is 2.3. Meanwhile, a peak is also found at $95 \mathrm{~Hz}$, where the amplification ratio can be up to 5 and the output stroke is nearly doubled. This could possibly provide mechanical advantages for the stage operated at this regime.

Meanwhile, both the input and the output stiffness are also examined. As shown in Fig. 10, for actuating frequency less than $80 \mathrm{~Hz}$, their average values are $98 \mathrm{~N} / \mathrm{mm}$ and 48 $\mathrm{N} / \mathrm{mm}$, respectively. Again there also exhibits a peak of stiffness at around $95 \mathrm{~Hz}$ where the stiffness is nearly 5 times larger than their corresponding nominal values at low frequency.

Finally, in order to determine the natural frequency of the stage, an essential modal analysis is conducted using hammer testing. By examining the power spectrum of the testing results, the natural frequency is determined to be 197 $\mathrm{Hz}$. Notice that this value is actually similar to our previous metallic compliant stage design $(\sim 250 \mathrm{~Hz})^{(12)}$. This demonstrating the possible advantage of the 3DP-based stage design. I.e., more compliant while the natural frequency is maintained in comparison with that made of engineering metallic alloys.

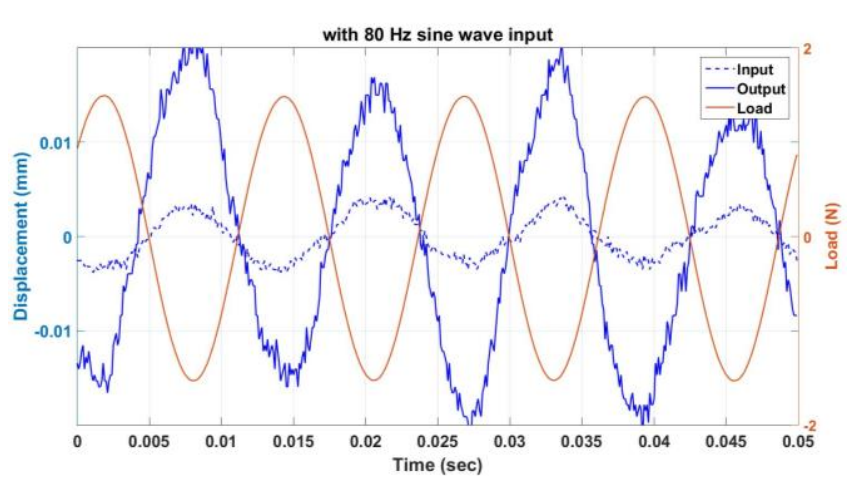

Fig. 8. Stage motion between displacements and load with a $0.3 \mathrm{~V}$ input.

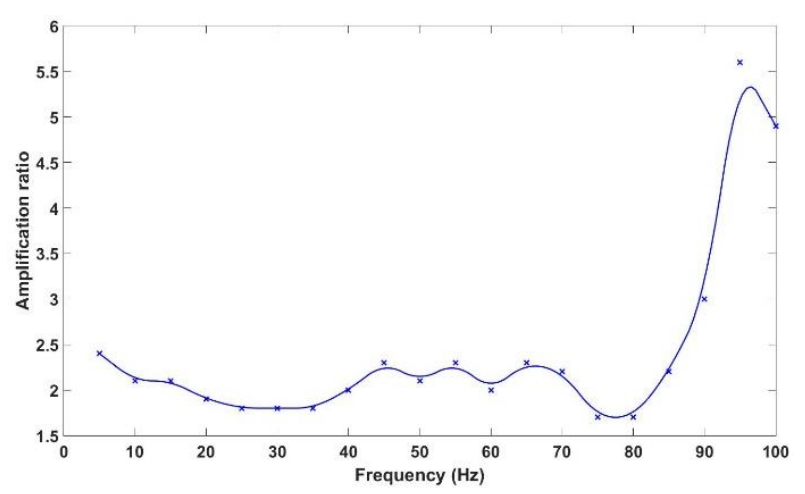

Fig. 9. Amplification experiment result. 


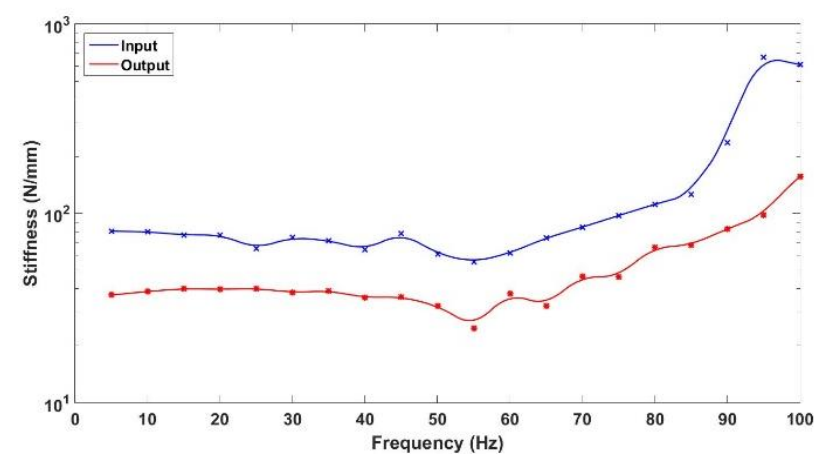

Fig. 10. Stiffness experiment results.

\section{Cantilever Beam test}

Before integrating rubber into the compliant stage for improving its damping capability, a fundamental study on the damping capability of such a composite structure should be investigated by using simple structure. Through the test, it is possible to quantize the amount of damping enhancement and the most efficient location for adding damping, which usually occurs at the maximum shear stress location by classic shear damping theory ${ }^{(5)}$. For beam bending with loading, this critical location usually occurs at the neutral axis schematically shown in Fig. 11. Therefore, in this work, beam-liked structures are fabricated for performing the investigation by using plastic 3DP and traditional metal machining.

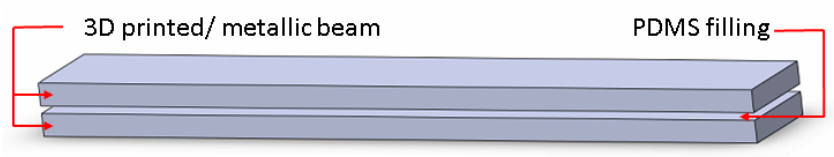

Fig. 11. Simple beam geometry used for damping characterization.

\subsection{Beam fabrication}

The cantilever beam fabricated using 3DP is designed and shown in Fig. 12. By creating cavities on the structure, it is possible to fill PDMS at specific locations. By subsequent vibration test, it is possible to correlate the PDMS filling parameters with the achieved damping for subsequent applications. Detail dimension of this ditchshape design is also shown in Fig. 13. The beam thickness is $7 \mathrm{~mm}$. In this study, the first four channels are filled with PDMS as the first case to be studied.

Meanwhile, for investigating the effect of inherent material damping and as a bridge for future metallic 3D printing design, aluminum cantilever beams with the same size of that fabricated by using 3DP are also fabricated and shown in Fig. 14. PDMS will also be filled in the core between two metallic structures (i.e., the neutral axis).

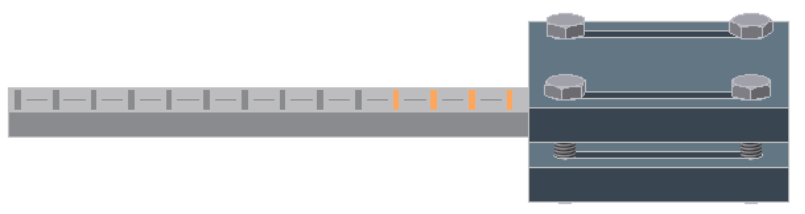

Fig. 12. Schematic plot of the 3D printed beam.

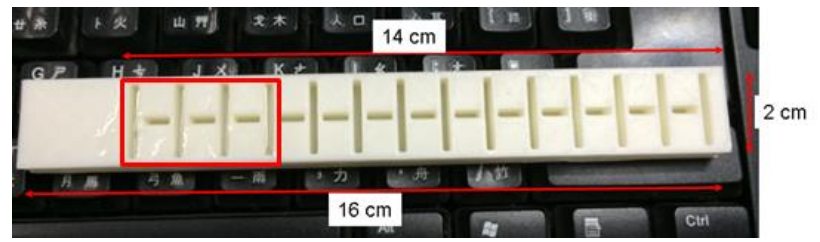

Fig. 13. The 3D printed beam.

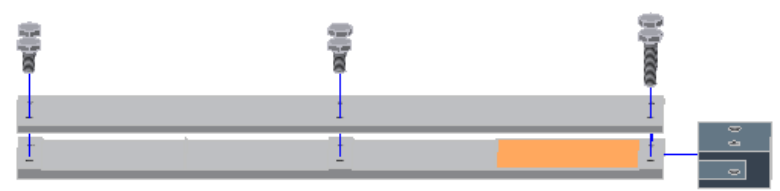

Fig. 14. Schematic plot of the aluminum beam.

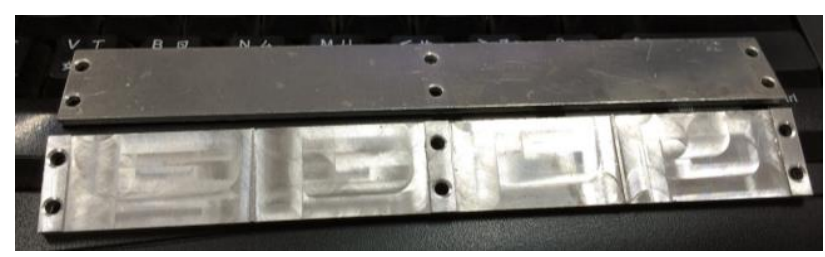

Fig. 15. The aluminum beam.

\subsection{Experiment demonstration}

As shown in Fig. 16, a vibration characterization system is setup by employing a shaker (MB MODAL50, peak force $=50$ pounds, stroke $=1$ inches ) and a function generator for providing frequency sweeping from near DC to approximately $160 \mathrm{~Hz}$. A MEMS accelerometer (EVAL ADXL326Z, gain $=51 \mathrm{mV} / \mathrm{g}$ ) is attached at the tip to measure the resulted acceleration. Both the spectrums before and after PDMS fillings are generated for quantitatively comparison and for identifying the amount of damping enhancement.

The test results are shown in Fig. 17 for aluminum and 3DP ABS beams, respectively. It can be seen that for aluminum beam has a higher natural frequency due to its larger Young's modulus and the quality factor decreases considerably after adding PDMS. This indicates the 
effectiveness of PDMS in damping enhancement. On the other hand, the 3DP ABS beam shows a lower natural frequency due to its low Young's modulus. The damping enhancement of adding PDMS is not significant since the material damping of ABS is comparable with that of PDMS.

In both cases, the natural frequencies are slightly increased (but not significant) due to the stiffness contributed by PDMS. The estimated natural frequency, damping ratio, and damping coefficients are listed in Table 2.

The above preliminary characterization results indicate that adding rubber should be helpful for improving system damping. However, it must be aware that the damping capability of the filling rubber should be much larger than the material damping of the original structure. This argument can be seen from the strong improved results obtained from the aluminum beam and the insignificant improvement from the 3DP ABS beam. Meanwhile, the filling location should also play an important role. Adding rubber filler at wrong place may not improve the damping and it actually increase the modal mass and results in a reduction in natural frequency.

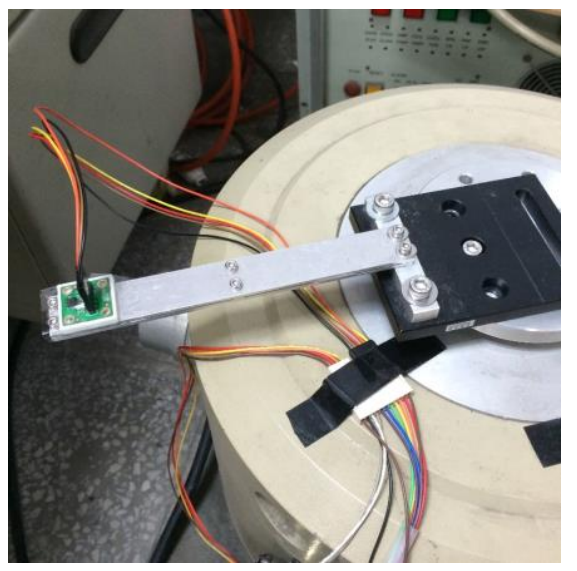

Fig. 16. Sweeping test setup of the beam.

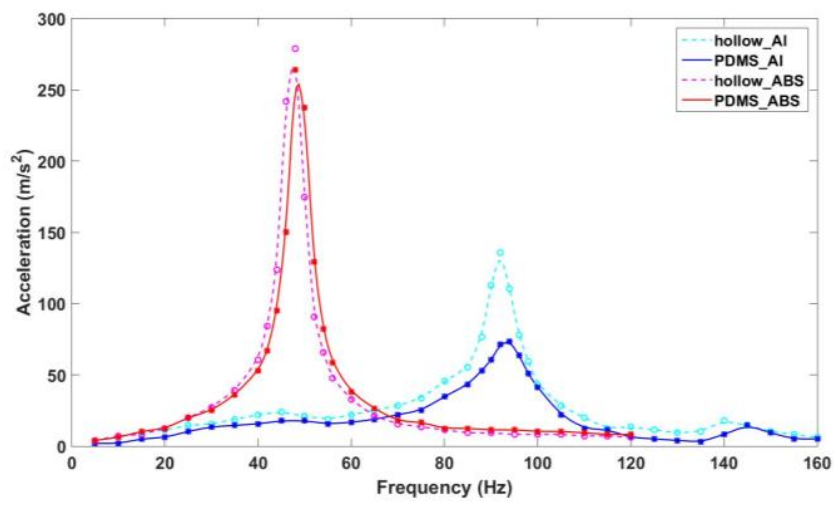

Fig. 17. Sweeping test result of aluminum and 3DP ABS beams.
Table 2. Summary of the damping from the beam tests.

\begin{tabular}{|c|c|c|c|}
\hline Hollow (Al) & $\begin{array}{c}\text { natural } \\
\text { frequency }(\mathrm{Hz})\end{array}$ & $\begin{array}{c}\text { damping } \\
\text { ratio }\end{array}$ & $\begin{array}{c}\text { damping } \\
\text { coefficient } \\
(\mathrm{N} \cdot \mathrm{s} / \mathrm{m})\end{array}$ \\
\hline PDMS (Al) & 92 & 0.034 & 0.654 \\
\hline Hollow (ABS) & 48 & 0.098 & 1.943 \\
\hline PDMS (ABS) & 49 & 0.05 & 0.108 \\
\hline
\end{tabular}

\section{Experiment demonstration of stage}

After finishing the beam characterization, the dynamic characterization of the compliant stage shown in Section 3 filled with PDMS is evaluated in this section. Spectrums of both without and with PDMS filling are characterized and compared. Fig. 18 shows the experimental setup and the PDMS-filled stage is shown in Fig. 4(b).

The characterized spectrums are shown in Fig. 19. It can be seen that the natural frequency of the PDMS-filled stage is reduced to its original value of $50 \mathrm{~Hz}$ to $38 \mathrm{~Hz}$. This is not surprising since the presence of PDMS effectively increases the modal mass and thus the natural frequency is reduced. The damping ratio and the damping coefficient, on the other hand, varies from 0.043 to 0.045 and $2.48 \mathrm{~N} \cdot \mathrm{s} / \mathrm{m}$ to $3.69 \mathrm{~N} \cdot \mathrm{s} / \mathrm{m}$. This indicates that the damping capability is slightly improved. Although the improvement is not significant, the concept is validated. In the future, with the metallic 3DP stage, the damping enhancement should be more obvious.

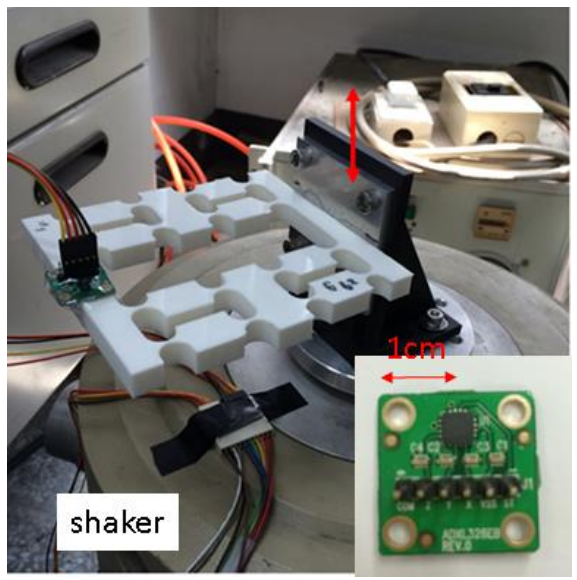

Fig. 18. Sweeping test setup of the stage. 


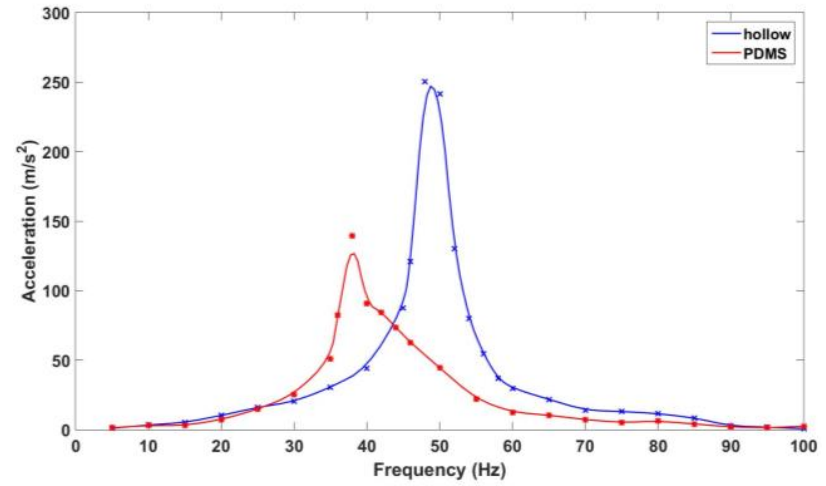

Fig. 19. Sweeping test results of the stage.

\section{Conclusion}

In this work, cantilever beam and a well-recognized compliant stage, served as the platform, is designed and realized by using plastic $3 \mathrm{D}$ printing and liquid PDMS casting for investigating the feasibility of improving the damping characteristics of compliant stage. Essential performance and dynamic tests are conducted to examine the functional performance and dynamic characteristics of the stage. The preliminary characterization results indicate that with proper design, the functional performance of the plastic stage can actually comparable with those fabricated by wire EDM metallic stages. Meanwhile, the enhancement of damping strongly depends on rubber injected position. Rubbers must be added in the portions where strong elastic deformations are expected for improving the damping capability. Based on the current results, it is expected the damping enhancement in real metallic 3DP could be more profound. Although primitive, the study shows promising results for continuing future more comprehensive studies in future stage designs using both plastic and metallic 3D printings.

\section{Acknowledgment}

This work is supported by the ministry of science and technology (MOST) of Taiwan under contract numbers MOST 104-2221-E-006-121-MY3, MOST 104-2221-E218-010 and industrial technology research institute through the cooperation project with NCKU.

\section{References}

(1) I. Her, and J. C. Chang., "A Linear Scheme for the Displacement Analysis of Micropositioning Stages with Flexure Hinges, ” ASME Trans., Journal of Mechanical Design, Vol.116, No.3, Sept.1994, pp.770-776.

(2) J. P. Davim, "Machining: Fundamentals and Recent Advances (Ed.)," Springer, 2008.

(3) M. Castilho, M. Dias, U. Gbureck, J. Groll, P. Fernandes, I. Pires, B. Gouveia, J. Rodrigues, E. Vorndran. Fabrication of computationally designed scaffolds by low temperature 3D printing. Biofabrication 2013; 5, published online.

(4) D. Benoit., "Computational Design of Lightweight Structures: Form Finding and Optimization," John Wiley \& Sons Inc, 2014.

(5) E. R. Marsh. "An Integrated Approach to Structural Damping," Doctor Thesis, Massachusetts Institute of Technology, May 1994

(6) Y. Li., Q. Xu., "Design and Analysis of a Totally Decoupled Flexure-Based XY Parallel Micromanipulator," IEEE Trans. Robot. Vol.25, No.3, pp.645-657. 2009.

(7) N. Lobontiu and E. Garcia, "Analytical model of displacement amplification and stiffness optimization for a class of flexure-based compliant mechanisms," Comput. Struct., vol. 81, no. 32, pp. 2797-2810, 2003.

(8) J. H. Kim, S. H. Kim, and Y. K. Kwak, "Development and optimization of 3-D bridge-type hinge mechanisms,' Sens. Actuators A, Phys., vol. 116, no. 3, pp. 530-538, 2004.

(9) K.-B. Choi and J. J. Lee, “Analysis and design of linear parallel compliant stage for ultra-precision motion based on 4-PP flexural joint mechanism," in Proc. Int. Conf. Smart Manuf. Appl., Gyeonggi-do,Korea, 2008, pp. 3538.

(10) S. S. Rao, "Mechanical Vibrations (5th ed.)," Upper Saddle River, N. J.: Prentice Hall, 2011.

(11) Y. Li., “A Novel Piezoactuated XY Stage With Parallel, Decoupled, and Stacked Flexure Structure for Micro/Nano positioning" IEEE Trans. Industrial Electronics. Vol. 58, No. 8. pp.3601-3614. 2011.

(12) J.W. Lee, "Design, Analysis, and Control of a Cascaded Piezoelectric Actuated Two-Degrees of Freedom Precision Stage", Master Thesis, Department of Mechanical Engineering, National Cheng Kung University, R.O.C., 2012. 